\title{
DEC 171990
}

Covariant Quark Model of Pion Structure

Hiroshi Ito, W. W. Buck

Department of Physics, Hampton University

Hampton, Virginis 23668, USA

Frane Gross

Physics Department, College of William and Mary

Williamsburg, VA 23185, USA

and

Continuous Electron Beam Accelerator Facility

12000 Jefferson Avenue, Newport News, VA 23606

\section{Continuous}

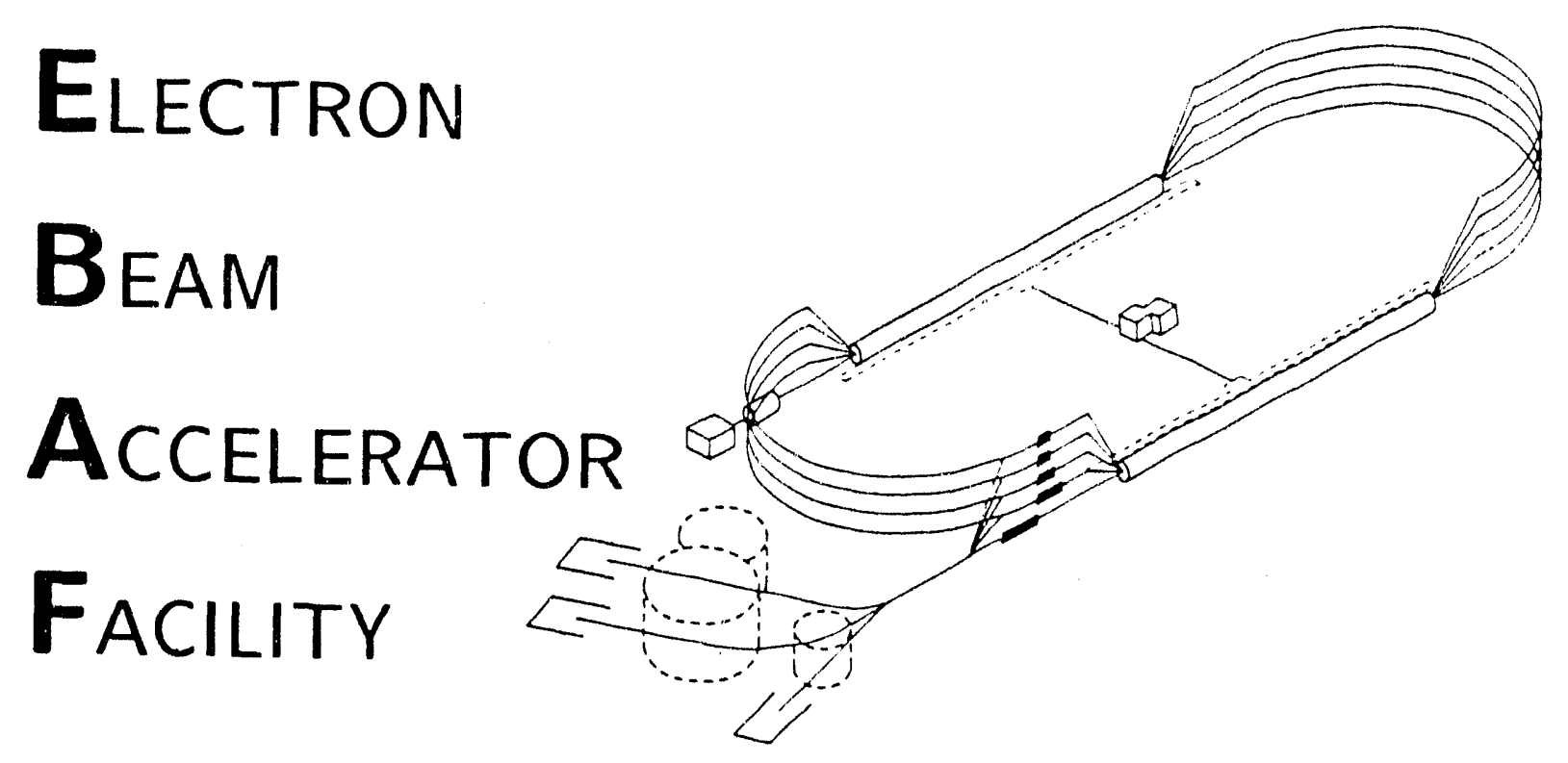

SURA SOUTHEASTERN UNIVERSITIES RESEARCH ASSOCIATION

\section{CEBAF}

Newport News, Virginia 
Copies available from:

\author{
Library \\ CEBAF \\ 12000 Jefferson Avenue \\ Newport News \\ Virginia 23606
}

The Southeastern Universities Research Association (SURA) operates the Continuous Electron Beam Accelerator Facility for the United States Department of Energy under contract DE-AC05-84ER40150.

\title{
DISCLAIMER
}

This report was prepared as an account of wcrk sponsored by the United States government. Neitber the United States nor the United States Department of Energy, nor any of their employees, makes any warranty, express or implied, or assumes any legal liability or responsibility for the accuracy, completeness, or usefulness of any information, apparatus, product, or process disclosed, or represents that its use would not infringe privately owned rights. Reference berein to any specific commercial product, process, or service by trade name, mark, manufacturer, or otberwise, does not necessarily constitute or imply its endorsement, recommendation, or fovoring by the United States government or any agency thereof. The views and opinions of authors expressed herein do not necessarily state or reflect those of the United States government or any agency thereof. 


\title{
Covariant Quark Model of Pion Structure
}

\author{
Hiroshi Ito, W. W. Buck \\ Department of Physics, Hampton University \\ Hampton, Virginia 23668, U.S.A. \\ Franz Gross \\ Physics Department, College of William and Mary \\ Williamsburg, Va. 23185, U.S.A. \\ and \\ Continuous Electron Beam Accelerator Facility \\ 12000 Jefferson Avenue, Newport News, Va. 23606
}

\begin{abstract}
A relativistic guark model wave function of the pion is obtained from a fit to the experimental data, including the charge radius, charge form factor and weak decay constant. The results are quite stable for different choices of quark mass. The two-photon decay width of the neutral pion and the asymptotic form factor for the $\gamma^{*}+\pi^{\circ} \rightarrow \gamma$ transition are estimated.
\end{abstract}


The structure of hadronic matter has been studied using a variety of theoretical techniques, the choice of which depends on the size of the system or the distance scales involved. Single hadrons have been described by giving their quark wave functions. The "hard" parts of these wave functions, for use at very high momentum transfer(short distance), can be calculated from perturbative QCD(PQCD)[1]. The "soft"parts, which describe the structure at long and intermediate distances, are modeled in a variety of ways[2-7]. Multi-nucleon systems have been successfully described by effective meson theories[8-9] based on relativistic dyamics, in which the nuclear force is described by the exchange of mesons. However, at short distances, when the nucleons overlap, it is not clear that processes involving the exchange of mesons can be separated from the quark structure of the nucleons (or mesons) themselves. To study such questions quantitatively, and to understand how(or where) effective meson theories break down, we need simple models of the quark structure of nucleons and mesons. Such models can be used, for example, to calculate the strong-form factors which arise at the meson-nucleon vertices. They also can be used to estimate both meson exchange and quark interchange processes in a consistent manner.

In this work we present a model of the "soft" part of the pion wave function intended for use in such a program. Instead of calculating the wave function from some specific dynamical model of the confining potential, we chose to model the wave function directly. We chose a fully covariant wave function of the Bethe-Salpeter type, with four adjustable parameters. These parameters are adjusted to fit the charge radius $\left(r_{n}^{2}\right)$, the low- $q^{2}$ region of the charge form factor $\left(F^{\pi}\left(q^{2}\right)\right)$ and weak deray constent $\left(f_{\pi}\right)$. The sensitivity of the andysis to the quark mass is examined. We estimate the width of two-photon decay $\left(\Gamma_{\pi_{0}} \rightarrow \gamma \gamma\right)$ by using the pion weve function so obtained, and the asymptotic form factor for the $\gamma^{*}+\pi^{\circ} \rightarrow \gamma$ transition in the $e+\pi^{\circ} \rightarrow e+\gamma$ reaction is presented for comparison with that given by PQCD.

Ous model wave function has two characteristic features: (1) The momentum dependence has a power-law behavior, with mass scales which can be adjusted to match the scale of different physical processes. We believe this is more realistic than the Gaussian functions frequently used. (2)The form is fully covariant, so that the wave functions can be boosted 
without any of the difficulties associated with non-relativistic models. In addition, angular momentum is conserved and the relativistic spin structure of the hadron is easily studied, which is not the case for light-cone wave functions. These features make it possible, in principle, to use these wave functions as part of a combin $d$ relativistic phenomenology in which long-range physics is described by a relativistic meson theory and the short-range physics is described by a relativistic quark model.

The calculation starts with the following ansatz for the covariant wave function of the pion.

$$
\begin{aligned}
\Psi(p, k) & =\frac{\mathcal{N}}{D\left(k^{2}\right)}\left[A \gamma^{5}+B \gamma^{5} p\right] \chi_{c} \chi_{f} \\
& =S\left(k+\frac{p}{2}\right) \Gamma(p, k) S\left(k-\frac{p}{2}\right)
\end{aligned}
$$

where $S(p)$ is the quark propagator with quark mass $m_{q}, \Gamma(p, k)$ the $\pi q \bar{q}$ vertex function, $p$ the four momentum of pion, and $k$ is the relative momentum of the $q \bar{q}$ pair. We use the matrix representation for the relativistic $q \bar{q}$ spin structure[10], and the pseudo-scalar property of the pion is represented by $\gamma^{5}$ and $\gamma^{5} p$ with a mixing parameter $(\eta) ; A \equiv 1-\eta$ and $B \equiv \frac{\eta}{M}\left(M c^{2}=138 . M e V\right.$ is used $)$. The momentum distribution of the $q \bar{q}$ pair is given by $D\left(k^{2}\right)$, for which the explicit functional form is given below. The overall normalization $(\mathcal{N})$ is determined by the charge normalization, and $\chi_{c}$ and $\chi_{f}$ are the color-and flavorwave functions ${ }^{\dagger}$ respectively.

The weak decay constant $\left(f_{\pi}\right)$ is given by the loop diagram(Fig.1), in which the $q \bar{q}$ pair couples to the weak current, $J_{W}^{\mu}=-i \tau^{+} \gamma^{\mu}\left(1-\gamma^{5}\right)$ with $\pi^{+}=\frac{1}{2}\left(\tau_{1}+i \tau_{2}\right)(\pi$ is the Pauli spin matrix). Therefore,

$$
\sqrt{2} f_{\pi} p^{\mu}=-2 \cdot\left\{\tau^{+} \chi_{f}^{\pi^{-}}\right\} \operatorname{Tr}\left\{\chi_{c}\right\} \int \frac{d^{4} k}{(2 \pi)^{4}} \operatorname{Tr}\left\{\gamma^{\mu}\left(1-\gamma^{5}\right) \Phi(p, k)\right\}
$$


where $\operatorname{Tr}\left\{\boldsymbol{\tau}^{+} \chi_{f}^{\pi^{-}}\right\}=1, \operatorname{Tr}\left\{\chi_{c}\right\}=\sqrt{n_{c}}\left(n_{c}=3\right)$ and $\Phi(p, k) \equiv \frac{\mathcal{N}}{D\left(k^{3}\right)}\left[A \gamma^{3}+B \gamma^{3} \not p\right]$. This gives

$$
f_{\pi}=\frac{4 B \sqrt{n_{c}}}{\sqrt{2}} \int \frac{d^{4} k}{(2 \pi)^{4}} \frac{\mathcal{N}}{D\left(k^{2}\right)}
$$

up to the absolute normalization $(\mathcal{N})$. If we chose $D\left(k^{2}\right)$ to behave like $\sim k^{n},(n>4)$, the integral in eq.(3) converges, and the asymptotic power counting of exclusive processes can be expres ied in terms of $f_{\pi}$. In Ref.5, a nonrelativistic Gaussian wave function boosted to the infinite momentum/light-cone frame is used to satisfy this condition, and the effect of perturbative gluon exchange is estimated. In this work, we choose a product of monopole functions, $D\left(k^{2}\right)=\prod_{i=1}^{3}\left[k^{2}-\Lambda_{i}^{2}\right]$, so that we can estimate the typical mass scales of the physical processes from the sizes of cutoff masses $\left(\Lambda_{i}\right)$ in the wave function. (A twoparameter model $D\left(k^{2}\right)=\left[k^{2}-\Lambda_{a}^{2}\right]\left[k^{2}-\Lambda_{b}^{2}\right]$, is also used for comparison.)

The low- $q^{2}$ region of the charge form factor, $F^{\pi}\left(q^{2}\right)$, can be obtained by the impulse diagram(Fig. 2 ) through the use of the soft wave function in eq.(1),

$$
F^{\pi}\left(q^{2}\right)\left(p^{\prime}+p\right)^{\mu}=M_{u}^{\mu}+M_{\frac{\mu}{d}}^{\mu}
$$

where the invariant amplitude for the u-quark process is given by

$$
M_{u}^{\mu}=-i \int \frac{d^{4} k}{(2 \pi)^{4}} \operatorname{Tr}\left\{\bar{\Psi}\left(p^{\prime}, k^{\prime}\right)\left(-i Q_{q} \gamma^{\mu}\right) \Psi(p, k) S\left(k-\frac{p}{2}\right)^{-1}\right\}
$$

Here $Q_{Q}$ is the quari charge operator giving $\operatorname{Tr}\left\{\chi_{f}^{\dagger} Q_{q} \chi_{f}\right\}=e_{u}(=2 / 3)$, for the $\pi^{+}$-case. The amplitude $\left(M_{\frac{\mu}{d}}\right)$ with the $\bar{d}$-quark coupling to the photon has the similar structure. Explicit calculation of $M_{u}$ and $M_{\bar{d}}$ reveals the gauge invariant feature of this exclusive 
process. The normalization $\mathcal{N}$ is determined by $F^{\pi}(0)=1$, and the charge radius is given by $r_{\pi}^{2}=-6 \lim _{q^{2} \rightarrow 0} \frac{d}{d q^{2}} F^{\pi}\left(q^{2}\right)$.

The analysis of our model wave function is performed with several different values of the quark mass $\left(m_{\bullet} c^{2}=10,100,300\right.$ and $\left.400 \mathrm{MeV}\right)$, by $\chi^{2}$-fitting to the static observables: $r_{\pi}=0.66 \pm 0.03 \mathrm{fm}, f_{\pi}=93 \pm 0.5 \mathrm{MeV}$ and the charge form factor in the low $-q^{2}$ region shown in the solid circles of Fig.3[11]. The results are presented in Table.1, in which one observes a stability under the change of the quark mass. This is due to the fact that the dependence on the quark mass exactly cancels with the normalization constant in the charge form factor: It can be shown that $F^{\star}\left(q^{2}\right)=\mathcal{N}^{2}\left[4 m_{q} A B+A^{2}+B^{2} m_{\pi}^{2}\right] f\left(q^{2}, \Lambda_{i}\right)$, where the quark mass $\left(m_{q}\right)$ does not appear in the momentum dependence, $f\left(q^{2}, \Lambda_{i}\right)$, therefore $\mathcal{N}^{2}=\left\{\left[4 m_{\mathrm{q}} A B+A^{2}+B^{2} m_{x}^{2}\right] f\left(q^{2}=0, \Lambda_{i}\right)\right\}^{-1}$. The weak decay constant,eq.(3), depends on the quark mass only through the normalization factor.

The second observation from our results is that the wave function with the cutoff masses of $\Lambda_{1} \sim 0.74 \mathrm{fm}^{-1}, \Lambda_{2} \sim 3.2 \mathrm{fm}^{-1}$ and $\Lambda_{3} \sim 3.6 \mathrm{fm}^{-1}$ reproduces the correct sizes of charge radius and charge form factor as well as the weak decay constant. (A similar result is obtained by the two-parameter model with $\Lambda_{a} \sim 0.65 \mathrm{fm}^{-1}$ and $\Lambda_{b} \sim 2.3 \mathrm{fm}^{-1}$; though, an extra cutoff, $\Lambda_{c} \sim 3-4 \mathrm{fm}^{-1}$, is needed to avoid the logarithmic divergence of the weak decay constant. This scale of the cut-off mass seems to be consistent with the NJL model $[3,4]$.) It is found that the charge radius and/or the low- $q^{2}$ behavior of charge form factor are contrclled by the smaller cutoff mass $\left(\Lambda_{1}\right)$ : A test analysis with the reference data of a "smaller pion" ( $r_{\pi}=0.4 \pm 0.05 \mathrm{fm}$ and $\left.f_{\pi}=93 \pm 0.5 \mathrm{MeV}\right)$ results in $\Lambda_{1}=1.46 \mathrm{fm}^{-1}, \Lambda_{2}=3.3 \mathrm{fm}^{-1}, \Lambda_{\mathrm{s}}=3.6 \mathrm{fm}^{-1}, \eta=0.09, f_{\pi}=92 \mathrm{MeV}$ and $r_{\pi}=0.42 \mathrm{fm}$. Vector meson dominance model is a successful hadronic description in the low energy domain. Vector meson process could be extracted by projecting the quantum numbers of rector mesons from the photon-quark vertex in Fig.2.

The two-photon decay width of the neutral pion, $\Gamma_{x^{\circ} \rightarrow \gamma \gamma}^{e x p}=7.57 \pm 0.3 \mathrm{eV}[12]$, has been evaluated with the triangle diagram[13] by assuming the point-like pion to be coupled to a fermion-antifermion pair. The importance of the axial anomaly has been extensively discussed[14]. It is tempting to estimate this observable by using our pion wave function, which reproduces the right size of the pion charge radius. The decay width is expressed 
by

$$
\Gamma_{\pi_{0 \rightarrow 2 \gamma \gamma}}=\frac{1}{32 \pi} \frac{1}{m_{\pi}} \sum_{\left(c_{c}, c_{b}\right)}\left|M\left(p, q_{a}, q_{b}\right)\right|^{2},
$$

Where the invariant amplitude is calculated from the triangle diagram (Fig.4),

$$
\begin{aligned}
M\left(p, q_{a}, q_{b}\right) & =i \operatorname{Tr}\left\{Q_{q} Q_{q} \chi_{f}^{\pi^{0}}\right\} \operatorname{Tr}\left\{\chi_{c}\right\} \\
& \times \int \frac{d^{4} k}{(2 \pi)^{4}} \operatorname{Tr}\left\{\left[\phi(b) S\left(k+\frac{p}{2}-q_{a}\right) \phi(a)+\phi(a) S\left(k+\frac{p}{2}-q_{b}\right) \phi(b)\right] \Phi(p, k)\right\} \\
& =-4 B i \sqrt{n_{c}}\left[\frac{e_{u}^{2}-e_{\frac{2}{d}}^{2}}{\sqrt{2}}\right] \epsilon^{\alpha \beta \mu \nu} \epsilon_{\alpha}(a) \epsilon_{\beta}(b) Z_{\mu} p_{\nu} .
\end{aligned}
$$

Here $\epsilon_{\alpha}(a), \epsilon_{\beta}(b)$ are the polarizations of the two photons $(a, b)$, and $\epsilon^{\alpha \beta \mu \nu}$ is the antisymmetric tensor. The integral $Z_{\mu}$ is given by

$$
Z_{\mu}=\int \frac{d^{4} k}{(2 \pi)^{4}} \frac{\mathcal{N}}{D\left(k^{2}\right)}\left[\frac{[k-q]_{\mu}}{(k-q)^{2}-m_{q}^{2}}-\frac{[k+q]_{\mu}}{(k+q)^{2}-m_{q}^{2}}\right]
$$

where $q \equiv \frac{1}{2}\left(g_{a}-q_{b}\right)$. The numerical results are presented in Table.1 for each quark mass. One observes the dependence on the quark mass, which is due to the quark propagation between the photon emissions in the diagram. A similar diagram provides the transition form factor for the $\gamma_{a}^{*}+\pi^{0} \rightarrow \gamma_{b}$ frocess, and this process has been recently considered in a nuclear target [15]. We evaluate the arymptotic behavior of this form factor to compare with the PQCD result up to the absciute uormalization. The form factor is defined[1] by 


$$
M^{\mu}=i e^{2} F_{\pi \gamma}\left(q_{a}^{2}\right) e^{\mu \nu \rho \sigma} p_{\nu} \epsilon_{\rho}(b) q_{\sigma}(a)
$$

where $p_{\nu}, q(a) \equiv q_{a}$ and $\epsilon(b)$ are the momenta of the real pion, a virtual photon(a) and the polarization vector of a real photon $(b)$ with momentum $q_{b}$ in the final state, respectively. The invariant amplitude is given by

$$
\begin{aligned}
M^{\mu} & =4 B i \operatorname{Tr}\left\{Q_{q} Q_{q} \chi_{f}^{\pi^{0}}\right\} \operatorname{Tr}\left\{\chi_{c}\right\} \epsilon^{\mu \nu \rho \sigma} p_{\nu} \epsilon_{\rho}(b) \\
& \times \int \frac{d^{4} k}{(2 \pi)^{4}} \frac{\mathcal{N}}{D\left(k^{2}\right)}\left[\frac{\left(k+\frac{p}{2}+q_{a}\right)_{\sigma}}{\left(\left[k+\frac{p}{2}+q_{a}\right]^{2}-m_{q}^{2}\right)}-\frac{\left(k+\frac{p}{2}-q_{b}\right)_{\sigma}}{\left(\left[k+\frac{p}{2}-q_{b}\right]^{2}-m_{q}^{2}\right)}\right] .
\end{aligned}
$$

The integral in eq.(11) becomes $\frac{4}{q_{a}^{2}}\left[\frac{p}{2}+q_{a}\right]_{\sigma} \int \frac{d^{4} k}{(2 \pi)^{4}} \frac{\mathcal{N}}{D\left(k^{2}\right)}$ in the $q_{a}^{2} \rightarrow \infty$ limit due to the rapid convergence of $D\left(k^{2}\right)$ and with the real photon condition $q_{b}^{2}=\left(p+q_{a}\right)^{2}=0$. We thus bave the asymptotic expression,

$$
\lim _{q_{a}^{2}-\infty} F_{\pi \gamma}\left(q_{a}^{2}\right)=\frac{\left[e_{u}^{2}-e_{\frac{2}{d}}\right]}{\sqrt{2}} \sqrt{n_{c}} \frac{16 B}{\varsigma_{a}^{2}} \int \frac{d^{4} k}{(2 \pi)^{4}} \frac{\mathcal{N}}{D\left(k^{2}\right)}
$$

By employing eq.(3), one finally obtains the expression,

$$
\lim _{q_{a}^{2}-\infty} F_{\pi \gamma}\left(q_{a}^{2}\right)=\frac{4}{3} \frac{f_{\pi}}{q_{a}^{2}}
$$

The results of our calculations can be summarized as follows:

(1) The pion charge radius and form factor in the low energy region $\left(Q^{2}<2 \mathrm{GeV}^{2} / \mathrm{c}^{2}\right)$ are well reproduced $\left(\chi^{2} /\right.$ data $\left.\sim 0.5\right)$ by our covariant quark model. The momentum distribution of the $q \bar{q}$ wave function is given by $D\left(k^{2}\right)=\prod_{i=1}^{3}\left[k^{2}-\Lambda_{i}^{2}\right]$ with $\Lambda_{1} \sim 0.74 \mathrm{fm}^{-1}, \Lambda_{2} \sim$ $3.2 \mathrm{fm}^{-1}$ and $\Lambda_{3} \sim 3.6 \mathrm{fm}^{-1}$. 
(2)Dependence on the quark mass is shown to be quite stable in the analysis, but more rapid dependence is found in the estimation of two-photon decay width,$\Gamma_{\pi_{0} \rightarrow \gamma \gamma}$.

(3)The asymptotic behavior of the $\gamma^{*}+\pi^{0} \rightarrow \gamma$ transition form factor is expressed in terms of $f_{\pi}$. This is a similar results to the one given by $\operatorname{PQCD}\left(F_{\pi \gamma}\left(q_{a}^{2}\right)=2 \frac{f_{q}}{q_{a}^{2}}\right)[1]$.

We conclude from the analysis that a covariant parametrization of the $q \bar{q}$ wave function provides a useful way to model the low-energy character of the pion. The asymptotic result for $\gamma^{*}+\pi^{\circ} \rightarrow \gamma$ form factor is, up to the absolute normalization, equivalent to PQCD.

A more complete model, including ail the possible spin structures[10] $\gamma^{5}, \gamma^{5} \not, \gamma^{5} \psi$, and

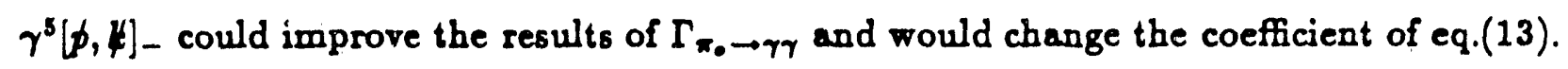
The effect of perturbative gluon exchange is calculated in a subsequent paper[16], which precucts results similar to those obtained in the light-cone frame[5]. Finally, we emphasize the strong advantage of using a covariant wave function for its clear connections to the relativistic field theory of hadrons.

The work of H.I was supported by NSF grant RII-8704038, that of W.W.B was supported by NASA grant NAG-1-447 and RII-8704038, and that of F.G was supported in part by the Department of Energy grant number DE-FG05-88ER40435. H.I and W.W.B would like to thank the Continuous Electron Beam Accelerator Facility(CEBAF) for the hospitality and for the computer facilities. 


\section{References}

[1] G. P. Lepage and S. J. Brodsky, Phys. Rev. D22 (1980) 2157.

[2] J. Gasser and H. Leutwyler, Nucl. Phys. B250 (1985) 465.

[3] Y. Nambu and G. Jona-Lasinio, Phys. Rev. 122 (1961) 345; 124 (1961) 246.

[4] V. Bernard, Phys. Rev. D34 (1986)1601

V. Bernard, Ulf G. Meissner and I. Zahed, Phys. Rev. D36 (1987) 819.

[5] N. Isgur and C. E. Llewellyn Smith, Phys. Rev. Lett. 52(1984) 1080.

[6] Z. Dziembowski, Phys. Rev. D37 (1988) 778,

O. C. Jacob and L. S. Kisslinger, Phys. Rev. Lett. 56 (1986) 225.

[ 7 ] P. L. Chung, F. Coester and W. N. Polyzou, Phys. Lett. B205 (1988) 545.

[8] F. Gross, Phys. Rev. 186 (1969) 1448; D10 (1974) 223.

[ 9 ] B. D. Serot and J. D. Walecka, Adv. in Nucl. Phys. 16, eds. J. W. Negele and E. Vogt (Plenum, New York, 1986).

[10] G. R. Farrar and D. R. Jackson, Phys. Rev. Lett. 43 (1979) 246.

[11] C. J. Bebek et al, Phys. Rev. D17 (1978) 1693.

[12] M. Aguilar-Benitez et al.(Particle Data Group), Phys. Lett. B170 (1986) 1.

[13] S. L. Adler, Phys. Rev. 177 (1969) $24 ? 6$.

J. S. Bell and R. Jackiw, Nuovo Cim. 60A (1969) 47.

[14] K. Fujikawa, Phys. Rev. Lett. 42 (1979) 1195.

[15] E. Hadjimichael and S. Fallieros, Phys. Rev. C39 (1989) 1438.

[ 16 ] H. Ito, W. W. Buck and F. Gross, submitted for publication. 


\section{Figure captions}

Fig. 1

The Feynman diagram for the weak decay of the pion. The solid loop and dotted line stand for the quark line and weak current, respectively.

Fig. 2

The impulse diagram of the pion charge form factor, where the wavy line denotes the virtual photon with momentum, $q$.

Fig. 3

The pion charge form factor $\left(Q^{2}=-q^{2}\right)$, where the data are taken from Ref.[11], and the solid circles are used in the fit. The solid line is the result of this analysis with $m_{q} c^{2}=300 \mathrm{MeV}$, and the parameters are given in the Table.1. The results with the two parameter model are shown for comparison, where the dashed line $\left(\Lambda_{a}=0.65 \mathrm{fm}^{-1}, \Lambda_{b}=\right.$ $\left.2.32 \mathrm{fm}^{-1}\right)$ and dotted-dash line $\left(\Lambda_{a}=0.90 \mathrm{fm}^{-1}, \Lambda_{b}=1.55 \mathrm{fm}^{-1}\right)$ are fitted to the charge radius $\left(r_{\pi}=0.66 \mathrm{fm}\right)$.

Fig. 4

Triangular diagrams of the two-photon decay process, where the wavy lines are real photons. 
Table captions

\section{Table 1}

The results of the present analysis, and the results for the two-photon decay width. 


\section{Foot notet}

$$
\chi_{f}^{\pi^{0}}=\left[\begin{array}{cc}
1 / \sqrt{2} & 0 \\
0 & -1 / \sqrt{2}
\end{array}\right], \quad \chi_{f}^{\pi^{+}}=\left[\begin{array}{ll}
0 & 1 \\
0 & 0
\end{array}\right], \quad \chi_{f}^{\pi^{-}}=\left[\begin{array}{ll}
0 & 0 \\
1 & 0
\end{array}\right] .
$$




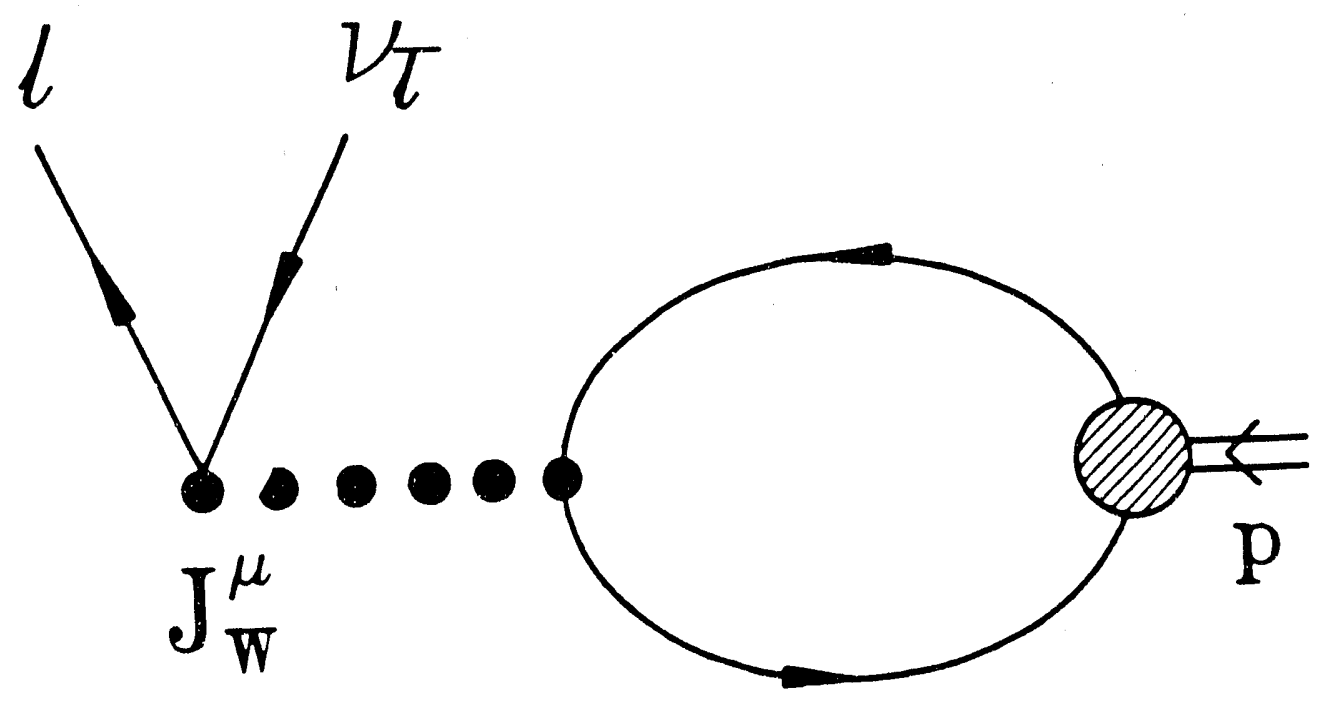

Fig. 1 


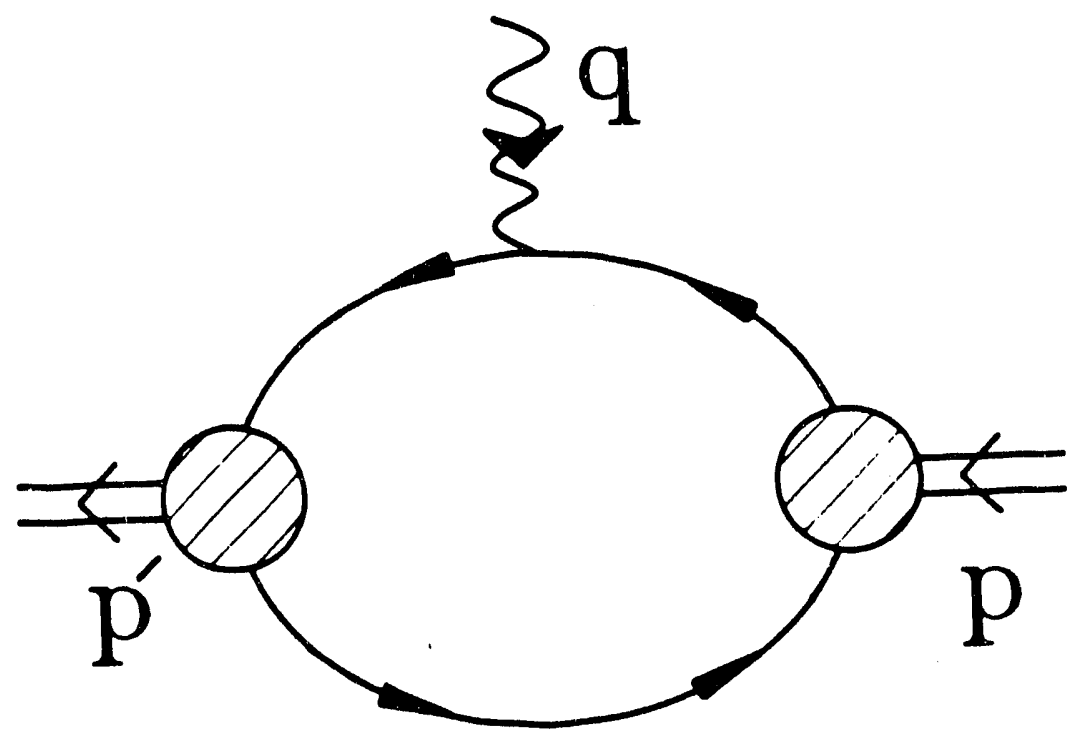

$I, \leq 2$ 


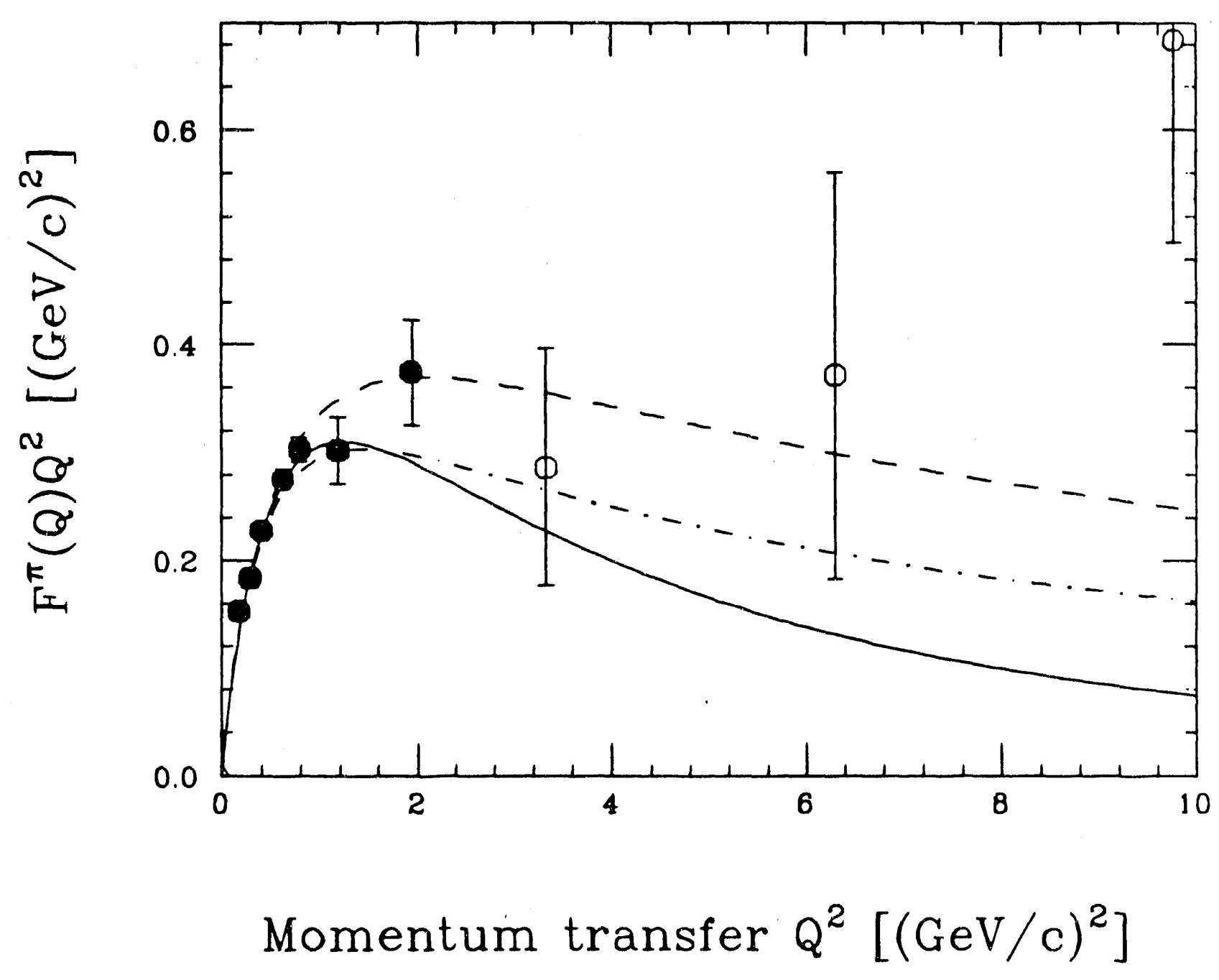

Fig 3 


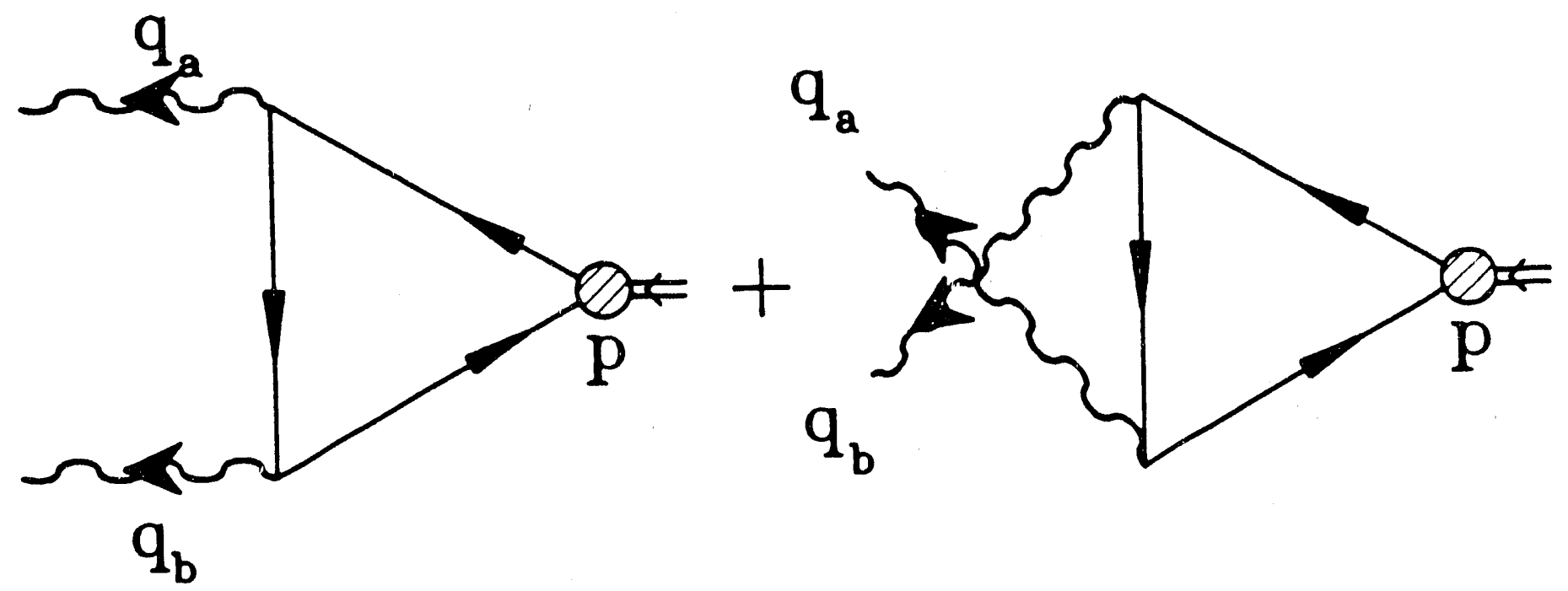

$F \because 4$ 


\begin{tabular}{|c|c|c|c|c|c|c|c|c|}
\hline$\left.m, c^{2} \mid M e^{\prime}\right\}$ & $\Lambda_{1}\left[f^{-1}\right]$ & $\Lambda_{2}\left[f m^{-1}\right]$ & $\Lambda_{3}\left[f m^{-1}\right]$ & $\eta$ & $r_{x}[f m]$ & $f_{\pi}[M e V]$ & $\chi^{2} /$ Data & $\Gamma_{\pi \cdot \rightarrow 2 \gamma}[\mathrm{eV}]$ \\
\hline 10 & 0.74 & 3.19 & 3.62 & 0.075 & 0.64 & 92.6 & 0.50 & 3.48 \\
\hline 100 & 0.74 & 3.18 & 3.68 & 0.083 & 0.64 & 92.8 & 0.52 & 2.85 \\
\hline 300 & 0.74 & 3.11 & 3.76 & 0.098 & 0.64 & 92.8 & 0.53 & 1.44 \\
\hline 400 & 0.74 & 3.12 & 3.60 & 0.112 & 0.64 & 82.8 & 0.54 & 1.11 \\
\hline
\end{tabular}



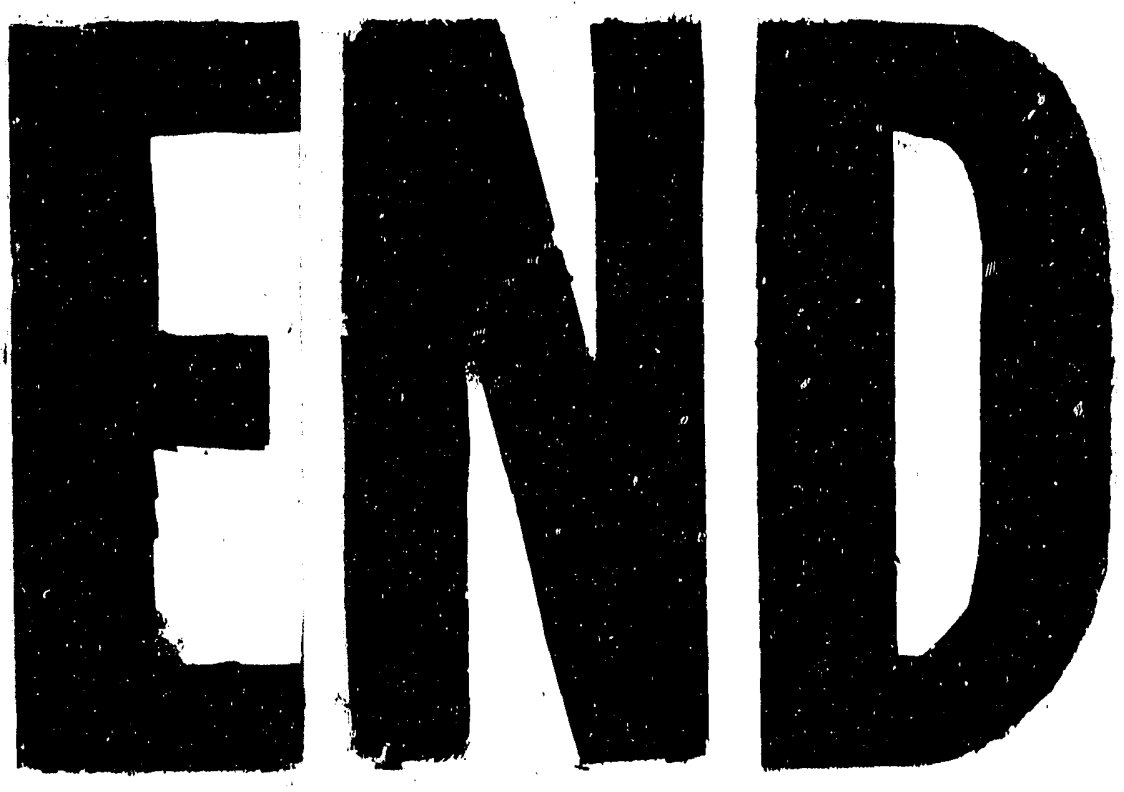

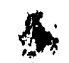
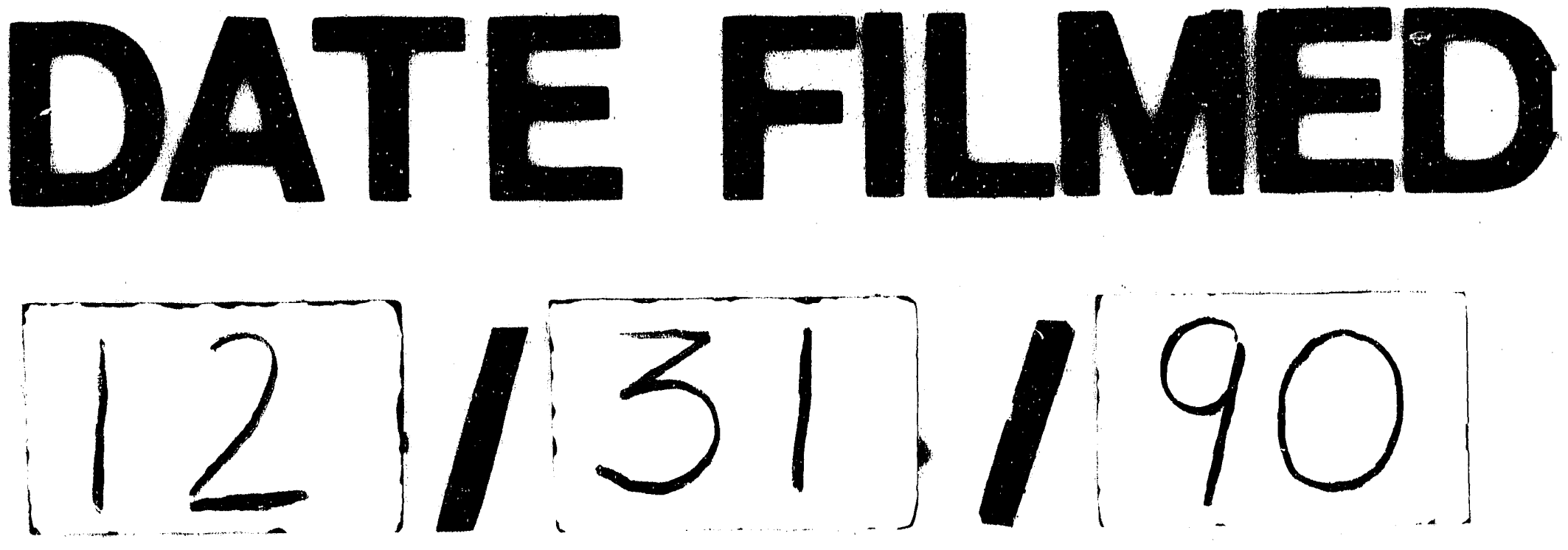
